\title{
EXPERIMENTAL PREDICTIONS FROM THE SUPERSTRING
}

E. Cohen, John Ellis, K. Enquist and D.V. Nanopoulos

$$
\text { CERN - Geneva }
$$

\section{A B S T R A C T}

We present phenomenological constraints on, and experimental predictions from, the minimal low energy model extracted from the ouperstring which appears able to generate the hierarchy $m_{W} \ll m_{P}$ (A) Quarks and charged leptons have specified couplings to a new neutral bos on $\mathrm{Z}_{\mathrm{E}}$ which must be heavier than the $\mathrm{Z}^{\circ}$. (B) Squark, slepton and gaugino masses are in the ratios

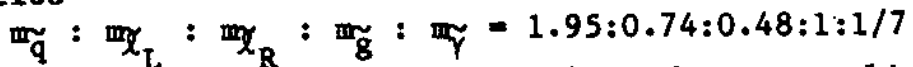

(C) Estimates are given of the Yukawa couplings which lead to $\mathrm{m}_{\mathrm{t}} \sim 55 \mathrm{GeV}$ and $\mathrm{m}_{\mathrm{W}}=\mathrm{m}_{\mathrm{p}} \exp \left(-0(1) / \alpha_{t}\right) \sim$ $80 \mathrm{GeV}$. (D) The absolute magnitudes of sparticle masses are estimated approximately. 
Many theorists take the superstring ${ }^{1), 2)}$ very serlously as a candidate Theory of Everything (TOE), but just how the theory makes contact with everyday, low energy, four-dimensional experiments is not yet completely clear. It is believed that the original 10-dimensional theory with its $\mathrm{E}_{8} \times \mathrm{E}_{8}$ ' gauge group reduces $^{3)}$ to a no-scale supergravity model in four dimensions ${ }^{4), 5)}$, with $E_{6}$ as a grand unification gauge group ${ }^{6}$. The low energy gauge group must ${ }^{6}$ ) be larger than $S U(3)_{C} \times S U(2)_{L} \times U(1)_{Y}$, containing at least one extra $U(1)$ gauge factor and possibly more low energy gauge bosons, depending on unknown details of the compactification from 10 to 4 dimensions. The number of fermion generations is likewise unclear ${ }^{7}$, though it is known $^{6)}$ that each one contains additional fields besides the conventional quarks and leptons. The masses of supersymmetric particles are determined by the scale of global supersymmetry breaking in the observable sector which should be $O\left(\mathrm{~m}_{W}\right)$. Although mechanisms exist 8$\left.), 9\right)$ for generating supersymmetry breaking from the hidden sector, feeding it through to the observable sector ${ }^{10}$ ) and determining its size dynamically through some variant of a no-scale model, the precise magnitudes of global supersymmetry breaking and of sparticle masses are not yet known. Thus superstring models offer a potential bonanza to experimentallsts - more gauge bosons, possibly more generations, additional particles besides quarks and leptons, as well as conventional sparticles - the problems are to decide how and where to prospect for them. A1though it may seem foolhardy and premature to attack these problems, we tackle them here in the mintmal low energy mode $1^{10}$ ) extracted from the superstring which appears able to generate the hierarchy $m_{W}<m_{p}$. Thus we assume a low energy $\mathrm{SU}(3)_{\mathrm{C}} \times \mathrm{SU}(2)_{\mathrm{L}} \times U(1)_{\mathrm{Y}} \times U(1)_{\mathrm{E}}$ gauge group with three quark and lepton generations and emphasize the rôle of an $\mathrm{SU}(3)_{C} \times \operatorname{SU}_{(2)} \times U(1){ }_{\mathrm{L}}$ singlet field $N$ coupled to the supersymmetric standard model Higgses $H, \vec{H}$. Our results are arranged in the order of increasing mode1-dependence.

(A) We first show that conventional quarks and charged leptons have well-defined vector and axial couplings to the new $z_{E}$ gauge boson. Low energy neutrino neutral current data 11$), 12$ ) force the $z_{E}$ to be significantly heavier than the $\mathrm{Z}^{\circ}$, whlle $\bar{p}$ p collider data ${ }^{13)}$ allow it to be almost degenerate with the $\mathrm{Z}^{\circ}: 90 \mathrm{GeV}<\mathrm{m}_{\mathrm{E}}<100 \mathrm{GeV}$ (which seems rather unlikely), or else weigh above $110 \mathrm{GeV}$.

(B) The ratios of soft supersymmetry breaking squark, slepton and gaugino masses are calculated from renormalization effects ${ }^{14}$ ): 


$$
m \tilde{q}^{\prime}: m_{\tilde{\ell}_{L}}: m_{\tilde{\ell}_{R}}: m_{\tilde{g}}: m_{\tilde{\gamma}}=1.95: 0.70: 0.38: 1: 1 / 7
$$

(C) Including the more model-dependent effects of $D$ term contributions, which are not negligible in this class of models, the results (1.a) are modified in one plausible variant to :

$$
m \tilde{q}: m_{\tilde{l}_{L}}: m \tilde{\ell}_{R}: m \tilde{g}: m \tilde{\gamma}=1.95: 0.74: 0.48: 1: 1 / 7
$$

Therefore, using $\mathrm{m}_{\mathrm{e}_{\mathrm{R}}} 223 \mathrm{GeV}$ from fruitless $\mathrm{e}^{+} \mathrm{e}^{-}$searches ${ }^{15)}$ and/or $\mathrm{m}_{\mathrm{g}} 240 \mathrm{GeV}$ from fruitless $\bar{p}$ p searches ${ }^{16)}$, we infer

$$
m_{\tilde{q}} \geqslant 93 \mathrm{GeV}, m_{\tilde{\ell_{L}}} \geqslant 35 \mathrm{GeV}, m_{\tilde{g}} \geqslant 48 \mathrm{GeV}, m_{\tilde{\gamma}} \geqslant 6.9 \mathrm{GeV}
$$

Renormalization calculations also demonstrate that weak gauge symmetry breaking is possible at a scale $\mathrm{v}, \overline{\mathrm{v}}, \mathrm{x} \equiv\langle 0|\mathrm{H}, \overline{\mathrm{H}}, \mathrm{N}| 0\rangle=0(200) \mathrm{GeV}: \mathrm{m}_{\mathrm{W}} \sim 80 \mathrm{GeV}$ if the $\mathrm{t}$ quark and $\mathrm{H} \overline{\mathrm{H}} \mathrm{N}$ Yukawa couplings are chosen :

$$
\lambda^{2}\left(m_{w}\right)=0.078, \lambda_{t}^{2}\left(m_{w}\right)=0.12
$$

The $Z_{E}$ boson is too light in this minimal model, but not all important effects have yet been included, and we regard these calculations only as an illustrative existence proof.

(D) Uncertainties in the minimization of the effective potential do not yet permit a precise determination of the global supersymmetry breaking scale, but we estimate it as

$$
m_{1 / 2} \simeq \theta(2) m_{W}
$$

with other sparticle masses scaled according to equation (1).

To discuss the additional neutral currents expected at low energles, it is

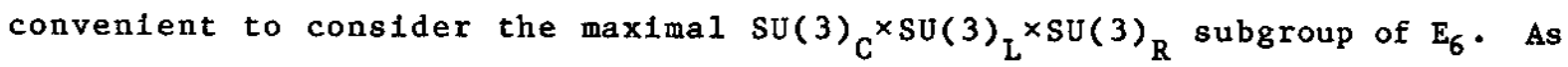
pointed out in Ref. 6), if one wishes to keep $\operatorname{SU}(3)_{\mathrm{C}} \times \mathrm{SU}_{(2)_{\mathrm{L}} \times \mathrm{U}_{(1)}}$ in the low energy gauge group, then since $Y=\frac{1}{2}\left(Y_{L}+Y_{R}\right): Y_{L} \equiv \operatorname{diag}(1 / 3,1 / 3,-2 / 3)_{L}$ and $Y_{R}=\operatorname{diag}(4 / 3,-2 / 3,-2 / 3)_{R}$, one cannot avold keeping both $Y_{L}$ and $Y_{R}$ unbroken down to low energies. The low energy group may be larger, but $\operatorname{SU}(3)_{C} \times S U(2){ }_{L} \times$ ${ }^{U(1)}{ }_{Y_{L}} \times U(1)_{Y_{R}}$ is the minimum possible extension of the standard model at low 
energies in the superstring. It is convenient to work with $Y$ and the orthogonal combination

$$
Y_{E}=\sqrt{\frac{3}{5}}\left(Y_{L}-\frac{1}{4} Y_{R}\right)
$$

where we have introduced a normalization façtor ensuring that the gauge coupling constant $g_{\mathrm{F}}$ associated with $\mathrm{Y}_{\mathrm{E}}$ is equal to $\mathrm{g}_{2}$ and $\mathrm{g}_{3}$ in the $\mathrm{E}_{6}$ symmetry limit. A simple renormalization group calculation gives

$$
\alpha_{E} \equiv g_{E}^{2} / 4 \pi=0.0164
$$

at scales $\lesssim \mathrm{m}_{\mathrm{W}}$. Using the definition (5) we find the following $\mathrm{Y}_{\mathrm{E}}$ assignments for conventional quarks and leptons $\left(q, q^{c}, l, l^{c}\right)$, the Higgses $(\vec{H}, H)$, the additional charge $-1 / 3$ quarks $\left(g, g^{c}\right)$ and the two $S U(3)_{C} \times \operatorname{SU}^{(2)} \times{ }_{L} \times(1)_{Y}$ singlet and neutral fields $\left(\mathrm{N}, \mathrm{N}^{\prime}\right)$ appearing in each $\underline{27}$ of $\mathrm{E}_{6}$ :

$$
\begin{gathered}
(u, d)_{L} u_{L}^{c} d_{L}^{c}\left(\nu, e^{-}\right)_{L} e_{L}^{c} \bar{H} H g g^{c} N N^{\prime} \\
Y_{E}=\sqrt{\frac{3}{5}} \times\left(\frac{1}{3}, \frac{1}{3},-\frac{1}{6},-\frac{1}{6}, \frac{1}{3},-\frac{1}{6},-\frac{2}{3},-\frac{2}{3},-\frac{1}{6}, \frac{5}{6}, \frac{5}{6}\right)^{(6 . b)}
\end{gathered}
$$

Equations (6.a) and (6.b) together specify completely the new neutral current couplings of the gauge boson $Z_{E}$ coupled to $Y_{E}$. Hence we are now in a position to calculate the effects of $Z_{E}$ exchange on low energy neutral current processes.

The most precise pieces of information about low energy neutral currents come from neutrino interactions, parity violation in ep scattering and in atoms ${ }^{11}$, and the forward-backward asymmetries in $\mathrm{e}^{+} \mathrm{e}^{-}$collisions ${ }^{12)}$. Table 1 shows $Z_{E}$ effects on neutrino neutral current parameters 11 ) and on the $\mathrm{e}^{+} \mathrm{e}^{-} \rightarrow \ell^{+} \ell^{-}$ forward-backward asymmetries ${ }^{12}$ ). Also shown are the experimental values ${ }^{11)}$ of these quantities : note that the $\mathrm{e}^{+} \mathrm{e}^{-}$constraints ${ }^{12)}$ are less important: although not exhibited, bounds from parity violation are similar. Taking $\sin ^{2} \Theta_{\mathrm{W}}$ $=0.227 \pm 0.010$ from $\mathrm{m}_{W}$ measured at the $\vec{p} p$ collider ${ }^{13}$ ) (as discussed later, the minimal superstring spectrum is consistent with $\sin ^{2} \Theta_{W}=0.21$ in leading order), we deduce from the low energy neutral current data that 


$$
y \equiv\left(g_{E}^{2} / g_{2}^{2}\right)\left(m_{W}^{2} / m_{Z_{E}}^{2}\right)=-0.12 \pm 0.24
$$

and hence, allowing 1 or $2 \sigma$ and using the value (6.a) for $g_{\mathrm{E}}^{2}$ :

$$
m_{Z_{E}}>(1.6,0.9) m_{W} \simeq(130,70) \mathrm{GeV}
$$

Next we turn to $\bar{p}$ collider production of the $z^{\circ}$ and $z_{E}$. It is easy to check using Equations $(6 . a)$ and $(6 . b)$ that for equal masses

$$
\sigma_{z_{E}} / \sigma_{z^{\circ}} \simeq 0.22 \pm 0.02
$$

The luminosity for $\bar{q} q \rightarrow Z_{E}$ collisions at the present $\bar{p} p$ collider can be described ${ }^{17)}$ to better than $10 \%$ accuracy in the mass range $70 \mathrm{GeV}<\mathrm{m}_{\mathrm{Z}}<110 \mathrm{GeV}$ by the formula

$$
\exp (-0.068 \Delta m) \times \mathscr{L}_{z^{0}}
$$

where $\Delta \mathrm{m}=\mathrm{m}_{\mathrm{Z}_{\mathrm{E}}}-\mathrm{m}_{\mathrm{Z}}$. If the $\mathrm{Z}_{\mathrm{E}}$ can decay freely 1nto all three generations of conventional quarks and leptons and also into three generations of $\mathrm{N}$ scalars (neons) and their $\tilde{N}$ fermion partners (ninos), then

$$
B_{E}\left(z_{E} \rightarrow e^{+} e^{-}\right) \simeq 1 / 108
$$

to be compared with the conventional $B_{0}\left(Z^{0} \rightarrow \mathrm{e}^{+} \mathrm{e}^{-}\right) \approx 3 \%$. Formula (11) may underestimate $B_{E}$, but using 1 t as a lower bound we combine 1 with (9) and (10) to find

$$
\sigma_{E} B_{E} / \sigma_{0} B_{0} \quad z \quad 0.02
$$

for $70 \mathrm{GeV}<m_{Z_{E}}<110 \mathrm{GeV}$. All high-mass $\ell^{+} \ell^{-}$palrs seen by the UAl and UA2 collaborations ${ }^{13}$ are compatible with the conventional $Z^{\circ}$. Requiring less than one event in each of the channels $z_{E} \rightarrow e^{+} e^{-}, \mu^{+} \mu^{-}$(UA1) and $z_{E}+e^{+} e^{-}$(UA2), we find that

$$
m_{Z_{E}}>110 \mathrm{GeV}
$$


Is allowed. There is one caveat to this analysis: if $\mathrm{m}_{\mathrm{Z}_{\mathrm{E}}} \sim \mathrm{m}_{\mathrm{Z}}$. then a few $Z_{E} \rightarrow \ell^{+} \ell^{-}$events could be hiding under the observed $Z^{\circ}$ peaks if

$$
90 \mathrm{GeV}<m_{Z_{E}}<100 \mathrm{GeV}
$$

Equations (8), (13) and (14) summarize the allowed ranges for $\mathrm{m}_{\mathrm{Z}}$.

In the above analyses we have ignored $\mathrm{Z}_{\mathrm{E}}-\mathrm{Z}^{\circ}$ mixing. We now examine this assumption a posteriori, and show that if the diagonal entries in the $\left(Z^{\circ}, Z_{E}\right)$ mass matrix were equal, the off-diagonal entries would push them far apart for representative values of the Higgs vacuum expectation values, so that the approximate degeneracy (14) is a highly unlikely scenario. The $\left(Z^{\circ}, Z_{E}\right)$ mass squared matrix is

$$
m_{z}^{2}\left[\begin{array}{cc}
1 & \sin \theta_{w} \frac{4 v^{2}-\bar{v}^{2}}{6\left(v^{2}+\bar{v}^{2}\right)} \\
\sin \theta_{w} \frac{4 v^{2}-\bar{v}^{2}}{6\left(v^{2}+\bar{v}^{2}\right)} & \sin ^{2} \theta_{w} \frac{4 v^{2}+\bar{v}^{2}+25 x^{2}}{9\left(v^{2}+\bar{v}^{2}\right)}
\end{array}\right]
$$

Using the relations (6) we find that in the limit of small off-diagonal elements relative to the difference in the diagonal elements (small mixing), the physical $\mathrm{Z}^{\circ}$ boson is shifted :

$$
m_{z}^{2}\left[1+\frac{\frac{1}{36} \sin ^{2} \theta_{w}\left(\frac{4 v^{2}-\bar{v}^{2}}{v^{2}+\bar{v}^{2}}\right)^{2}}{1-\frac{1}{9} \sin ^{2} \theta_{w} \frac{25 x^{2}+4 v^{2}+\bar{v}^{2}}{v^{2}+\bar{v}^{2}}}\right]
$$

as is the phystcal $Z_{E}$ boson : 


$$
m_{z E}^{2}=m_{z}^{2}\left[\frac{1}{9} \sin ^{2} \theta_{w} \frac{25 x^{2}+4 v^{2}+\bar{v}^{2}}{v^{2}+\bar{v}^{2}}-\frac{\frac{1}{36} \sin ^{2} \theta_{w}\left(\frac{4 v^{2}-\bar{v}^{2}}{v^{2}+\bar{v}^{2}}\right)^{2}}{1-\frac{1}{9} \sin ^{2} \theta_{w} \frac{25 x^{2}+4 v^{2}+\bar{v}^{2}}{v^{2}+v^{2}}}\right]
$$

On the other hand, if the diagonal entries in (15) are equal (maximal mixing), the splitting between the two mass eigenstates is

$$
\Delta m_{z} \simeq \frac{1}{6} m_{z} \sin \theta_{w} \frac{4 v^{2}-\bar{v}^{2}}{v^{2}+\bar{v}^{2}}
$$

For typical values of $\mathrm{v}$ and $\overline{\mathrm{v}}$ to be discussed later : $\mathrm{v} / \overline{\mathrm{v}} \sim 5$, the splitting (18) is impossibly large. We cannot exclude the possibility that in a different version of the mode1

$$
\frac{4 v^{2}-\bar{v}^{2}}{v^{2}+\bar{v}^{2}}<0.7
$$

so that $\Delta \mathrm{m}_{\mathrm{Z}}<5 \mathrm{GeV}$, in which case the possibility (already mentioned in our analysis of the $\bar{p}$ p collider data) of approximate degeneracy between the $z^{\circ}$ and the $Z_{E}$ cannot be ruled out. It will be interesting to see if future, more detailed, experiments find a split $Z^{\circ}$, although we regard the possibility as fortuitous and highly unlikely.

We now analyze the renormalization group equations of the low energy theory and derive the results (1) for the supersymmetry breaking mass parameters. It is easy to check that with the low energy particle content of three generations (6.b), in one-loop order

$$
\alpha_{3}=\text { const. } \alpha_{2}=\frac{\alpha_{3}}{1+\frac{3}{2 \pi} \alpha_{3} t}, \alpha_{1}=\alpha_{\epsilon}=\frac{\alpha_{3}}{1+\frac{9}{2 \pi} \alpha_{3} t}
$$

where $t=\ln \left(m_{x} / \mu\right)$. Therefore the GUT scale $m_{x}$ is given by 


$$
t_{x}=\ln \frac{m_{x}}{m_{w}}=\frac{2 \pi}{3 \alpha_{3}}\left[\frac{\alpha_{3} \sin ^{2} \theta_{w}}{\alpha_{e m}}-1\right]
$$

and with $\alpha_{e m}^{-1}=128$ and $\alpha_{3}=0.11$, this gives $\sin ^{2} \Theta_{W}=0.207$ and $t_{X}=36.2$ or $\mathrm{m}_{\mathrm{X}}=4.4 \times 10^{17} \mathrm{GeV}$, which is acceptable given the uncertainties of thresholds, two-loop effects, etc. The gaugino masses are in the ratios of the $\alpha_{1}(20)$ as usual :

$$
m_{\tilde{g}}: m_{\tilde{W}}: m_{\tilde{B}}: m_{\tilde{Z}_{E}}: m_{1 / 2}=1: 0.34: 0.15: 0.15: 1
$$

where $\mathrm{m}_{\frac{1}{2}}$ is the primordial supersymmetry breaking gaugino mass, which $1 \mathrm{~s}^{10)}$ the primary source of supersymmetry breaking. Assuming that third generation Yukawa couplings are the largest, the most important parts of the low energy scalar potential are derived from the superpotential

$$
W=\lambda N H \bar{H}+\lambda_{t} U Q H
$$

with the soft supersymmetry breaking terms

$$
V_{\text {soft }}=2 \lambda A \operatorname{Re} N H \bar{H}+2 \lambda_{t} A_{t} \operatorname{Re} U Q H+m_{N}^{2}|N|^{2}+m_{H}^{2}|H|^{2}+m_{H}^{2}|\bar{H}|^{2}(24)
$$

which obey the renormalization group equations

$$
\begin{aligned}
8 \pi^{2} \frac{d A}{d t} & =4 \lambda^{2} A+3 \lambda_{t}^{2} A_{t}+\left(3 g_{2}^{4}+2 g_{1}^{4}\right) m_{1 / 2} g_{3}^{-2} \\
8 \pi^{2} \frac{d A}{d t} & =\lambda^{2} A+6 \lambda_{t}^{2} A_{t}+\frac{16}{3} g_{3}^{2} m_{1 / 2}+\left(3 g_{2}^{4}+\frac{5}{3} g_{1}^{4}\right) m_{1 / 2} g_{3}^{-2} \\
-8 \pi^{2} \frac{d m_{N}^{2}}{d t} & =\frac{5}{3} g_{1}^{6} m_{1 / 2}^{2} g_{3}^{-4}-2 \lambda^{2}\left(m_{N}^{2}+m_{H}^{2}+m_{H}^{2}+A^{2}\right) \\
-8 \pi^{2} \frac{d m_{H}^{2}}{d t} & =\left(3 g_{2}^{6}+\frac{2}{3} g_{1}^{6}\right) m_{1 / 2}^{2} g_{3}^{-4}-\lambda^{2}\left(m_{N}^{2}+m_{H}^{2}+m_{H}^{2}+A^{2}\right)
\end{aligned}
$$




$$
\begin{aligned}
& -8 \pi^{2} \frac{d m_{H}^{2}}{d t}=-8 \pi^{2} \frac{d m_{H}^{2}}{d t}+g_{1}^{6} m_{1 / 2}^{2} g_{3}^{-4}-3 \lambda_{t}^{2}\left(m_{H}^{2}+m_{Q_{3}}^{2}+m_{U_{3}}^{2}+A_{t}^{2}\right) \\
& 8 \pi^{2} \frac{d \lambda}{d t}=\left(2 \lambda^{2}+\frac{3}{2} \lambda_{t}^{2}-\frac{3}{2} g_{2}^{2}-g_{1}^{2}\right) \lambda \\
& 8 \pi^{2} \frac{d \lambda_{t}}{d t}=\left(\frac{1}{2} \lambda^{2}+3 \lambda_{t}^{2}-\frac{8}{3} g_{3}^{2}-\frac{3}{2} g_{2}^{2}-\frac{5}{6} g_{1}^{2}\right) \lambda_{t}
\end{aligned}
$$

The squark and slepton masses are given by

$$
\begin{aligned}
& -8 \pi^{2} \frac{d m_{U_{3}}^{2}}{d t^{3}}=\left(\frac{16}{3} g_{3}^{6}+\frac{4}{3} g_{1}^{6}\right) m_{1 / 2}^{2} g_{3}^{-4}-2 \lambda_{t}^{2}\left(m_{H}^{2}+m_{U_{3}}^{2}+m_{Q_{3}}^{2}+A_{t}^{2}\right) \\
& -8 \pi^{2} \frac{d m_{D_{3}}^{2}}{d t}=\left(\frac{16}{3} g_{3}^{6}+\frac{1}{3} g_{1}^{6}\right) m_{1 / 2}^{2} g_{3}^{-4} \\
& -8 \pi^{2} \frac{d m_{Q_{3}}^{2}}{d t}=\left(\frac{16}{3} g_{3}^{b}+3 g_{2}^{b}+\frac{1}{3} g_{1}^{b}\right) m_{1 / 2}^{2} g_{3}^{-4}-\lambda_{t}^{2}\left(m_{H}^{2}+m_{v_{3}}^{2}+m_{Q_{3}}^{2}+A_{t}^{2}\right) \\
& -8 \pi^{2} \frac{d m_{1}^{2}}{d t}=\left(3 g_{2}^{6}+\frac{2}{3} g_{1}^{6}\right) m_{1 / 2}^{2} g_{3}^{-4} \\
& -8 \pi^{2} \frac{d m_{E}^{2}}{d t}=\frac{8}{3} g_{1}^{6} m_{1 / 2}^{2} g_{3}^{-4}
\end{aligned}
$$


representative values $\lambda_{t}^{2}\left(m_{X}\right)=0.0011$ and $\lambda^{2}\left(m_{X}\right)=0.02$ which correspond to $\lambda_{t}^{2}\left(m_{W}\right)=0.12, \lambda^{2}\left(m_{W}\right)=0.078$ and $m_{t} \sim 55 \mathrm{GeV}$, and also lead to weak gauge symmetry breaking at a realistic scale $\mathrm{m}_{W}=0\left(\mu_{0}\right)=0(100) \mathrm{GeV}$, as will be discussed shortly. The numerical predictions (1.a) are obtained directly from equation (22) and Table 2. In this class of mode1s, D-term contributions to scalar masses are not negligtble. We have evaluated these using the representative field vacuum expectation values (37), obtaining the results (1.b).

\section{Experimentally, we know}

$$
M_{L_{R}} \gtrsim 23 \mathrm{GeV}, M_{\tilde{g}} \geqslant 40 \mathrm{GeV}, m_{\tilde{q}} \geqslant 40 \mathrm{GeV}
$$

of which the first two are the most important constraints for the spectrum of equation (1.b). Inserting this into equation (1.b) we get the lower bounds on sparticle masses listed in equation (2). A posteriori, the lower bounds on $\left(m_{\mathrm{e}_{\mathrm{R}}}, \mathrm{m}_{\boldsymbol{\gamma}}\right.$ ) are at best marginally compatible with the MAC lower limits from a search for $e^{+} e^{-} \rightarrow \gamma+$ missing energy-momentum ${ }^{18)}$. We infer from equation (2) that $\mathrm{e}^{+} \mathrm{e}^{-}$experiments could well find single photons and possibly right-handed sleptons in the near future. The latter might also be detectable in $z^{\circ}$ decays at the $\overline{p p}$ collider, though left-handed sleptons might well not be accessible in either $W^{ \pm}$or $z^{\circ}$ decay. The $\bar{p} p$ collider may be able to see gluinos, but would be unlikely to see squarks according to equation (2).

Finally, we turn to the minimization of the effective potential, weak gauge symmetry breaking and the dynamical determination of the supersymmetry breaking scale $\mathrm{m}_{\frac{1}{2}}$. The tree-level low energy potential for $\mathrm{H}, \overline{\mathrm{H}}$ and $\mathrm{N}$ takes the following form for the real field values $v, \bar{v}, x$ :

$$
\begin{aligned}
V_{\text {cH }} & =m_{H}^{2} v^{2}+m_{H}^{2} \bar{v}^{2}+m_{N}^{2} x^{2}+2 \lambda A v \bar{v} x+\lambda^{2}\left(v^{2} \bar{v}^{2}+v^{2} x^{2}+\bar{v}^{2} x^{2}\right) \\
& +\frac{5}{24} g_{E}^{2}\left(x^{2}-\frac{4}{5} v^{2}-\frac{1}{5} \bar{v}^{2}\right)+\frac{1}{8}\left(g_{2}^{2}+g^{\prime 2}\right)\left(v^{2}-\bar{v}^{2}\right)^{2}
\end{aligned}
$$

As we bring the renormalization scale $\mu$ down from $\mathbb{R}_{X}$, the first squared mass parameter to go negative is $\mathrm{m}_{\mathrm{H}}^{2}$, which leads to a non-zero vacuum expectation value for $v$ : 
$-10-$

$$
v^{2}=\left|m_{H}^{2}\right| /\left[\frac{4}{15} g_{E}^{2}+\frac{1}{4}\left(g_{2}^{2}+g^{12}\right)\right]
$$

However, since ${ }^{10)}$

$$
m_{1 / 2}=-\frac{b g^{2}}{4}\left(\frac{1}{16 s_{R} T_{R}^{3}}\right)^{1 / 2} \lambda\left(\frac{v x}{\bar{v}}+\frac{\bar{v} x}{v}+\frac{v \bar{v}}{x}\right)
$$

we need all three of $v, \bar{v}, x \neq 0$ to get global supersymmetry breaking, and the renormalization group equations keep on evolving until the line $\bar{v}=\mathrm{x}=0$ becomes unstable. The trilinear term in (28) ensures that if one of $\bar{v}, x \neq 0$, then so also is the other. Instability sets in when

$$
\begin{aligned}
{\left[m_{N}^{2}\right.} & \left.+\left(\lambda^{2}-\frac{1}{3} g_{E}^{2}\right) v^{2}\right]\left[m_{H}^{2}+\left(\lambda^{2}+\frac{1}{15} g_{E}^{2}-\frac{1}{4}\left(g_{2}^{2}+g^{\prime 2}\right)\right) v^{2}\right] \\
& -\lambda^{2} A^{2} v^{2}<0
\end{aligned}
$$

at a renormalization scale $\mu_{0}=m_{\mathrm{P}} \exp \left(-0(1) / \lambda_{t}^{2}, \lambda^{2}\right): t_{0} \equiv \ln \left(m_{x} / \mu_{0}\right)$. In a no-scale mode $1^{5)}$, the global supersymmetry breaking scale $m_{\frac{1}{2}}(30)$ can be determined dynamically to be $O\left(\mu_{0}\right)$, as we now indicate. The full low energy effective potential is

$$
V=V_{\text {eff }}+\delta V: \delta V=\frac{1}{64 \pi^{2}} \operatorname{ser} m^{4}\left(\ln m^{2} / \mu^{2}-c\right)
$$

where $c$ is a renormalization-scheme-dependent constant ${ }^{*}$ ). Following Ref. 5), we choose $\mu: \delta \mathrm{V}=0$ which corresponds to

$$
\mu=\theta(1) m_{1} / 2
$$

With this choice of $\mu$,

*) Note that the naive cut-off-dependent $S t r M^{4} l n M^{2} / \Lambda^{2}$ is replaced ${ }^{19)}$ by $\mathrm{StrM}^{4} 1 \mathrm{nM}^{2} / \mu^{2}$ when a consistent renormalization prescription is adopted. Note also that the quadratically divergent $S t \mathrm{rM}^{2} \Lambda^{2}$ term can be absorbed in a renormalization of the parameters in the initial Kähler
potential 10$), 20)$. 


$$
V=V_{\text {eft }}(\mu)=e^{-4 t} P(t)
$$

where $P(t)$ is a polynomial of $t$ in the netghbourhood of the contour in $v, \bar{v}, x$ space $v_{\text {eff }}\left(\mu_{0}\right)=0$. The potential $v$ is therefore minimized with respect to $t$ when

$$
P(t)=\frac{1}{4} P^{\prime}(t): P^{\prime}=\theta\left(\frac{\alpha}{\pi}\right)
$$

Combining equations (33) and (35), we see that

$$
t=t_{0}+\theta(1)
$$

We therefore stop the renormalization group clock at a scale $\mu: t \equiv \ln \left(m_{X} / \mu\right)$ is given by the expression (36), and find for $\lambda_{t}^{2}\left(m_{W}\right)=0.12, \lambda^{2}\left(m_{W}\right)=0.078$

$$
\left(v^{2}, \bar{v}^{2}, x^{2}\right)=(0.81,0.04,0.24)
$$

Using $\frac{m_{W}^{2}}{W}=\frac{1}{2} g_{2}^{2}\left(v^{2}+\bar{v}^{2}\right)$ we can now calculate $m_{\frac{1}{2}}$ :

$$
m_{1 / 2}=\frac{\sqrt{2}}{g_{2}} m_{w}\left(\frac{m_{1 / 2}}{\sqrt{v^{2}+\bar{v}^{2}}}\right) \simeq 190 \mathrm{GeV}
$$

and hence the absolute values of the sparticle masses :

$$
m_{\tilde{q}}=360 \mathrm{GeV}, m_{\tilde{e}_{L}}=140 \mathrm{GeV}, m_{\tilde{e}_{R}}=90 \mathrm{GeV}, m_{\tilde{\nu}}=110 \mathrm{GeV}
$$

which are above the previous lower limits (2). Needless to say, the calculation of these absolute values involves many extra dynamical assumptions ${ }^{*}$ ) about the determination of field values in no-scale models which go beyond those used to obtain the ratio ( 1 ), and are sensitive to the mode1-dependent D-terms in particular. There could also be important contributions to the low energy effective potential beyond those discussed so far, for example from an extra

*) As emphasized in Ref. 10), the form (30) of $m_{1}$ implies $T_{R}=0(1)$ at the minimum. We have assumed that $S U(2) \times U(1)$ gauge ${ }^{2}$ breaking fixes $T_{R}$, though It is also possible ${ }^{21}$ that Planck mass dynamics fixes $T_{R}=0(1)$ [see also Binetruy and Gaillard, Ref. 20)] in which case the minimization of the potential could give somewhat different results, as we will discuss in a future publication. 
$\underline{27}+\underline{27}$ in addition to three generations like (6.b), or from additional low energy gauge interactions. Moreover, the third generation $N^{\prime}$ field in (6.b) may be important, as could be the first two generations of $N$ flelds. We actually need some modification to the above analysis, since the vacuum expectation values (37) would give $\mathrm{m}_{\mathrm{Z}} \otimes 40 \mathrm{GeV}$ when substituted into equation (17), which conflicts with the allowed range (13), (14). Many of the above effects would tend to increase $\mathrm{m}_{\mathrm{Z}}$, but the effect on the sparticle mass scale is unclear. However, given our dynamical assumptions the values (39) of the sparticle masses and of the $Z_{E}$ mass could be correct to within a factor $O(2)$.

\section{ACKNOWLEDGEMENTS}

We thank Fabio Zwirner for a corrective check of our results. One of us (K.E.) wishes to thank the Academy of Finland for financlal support. 


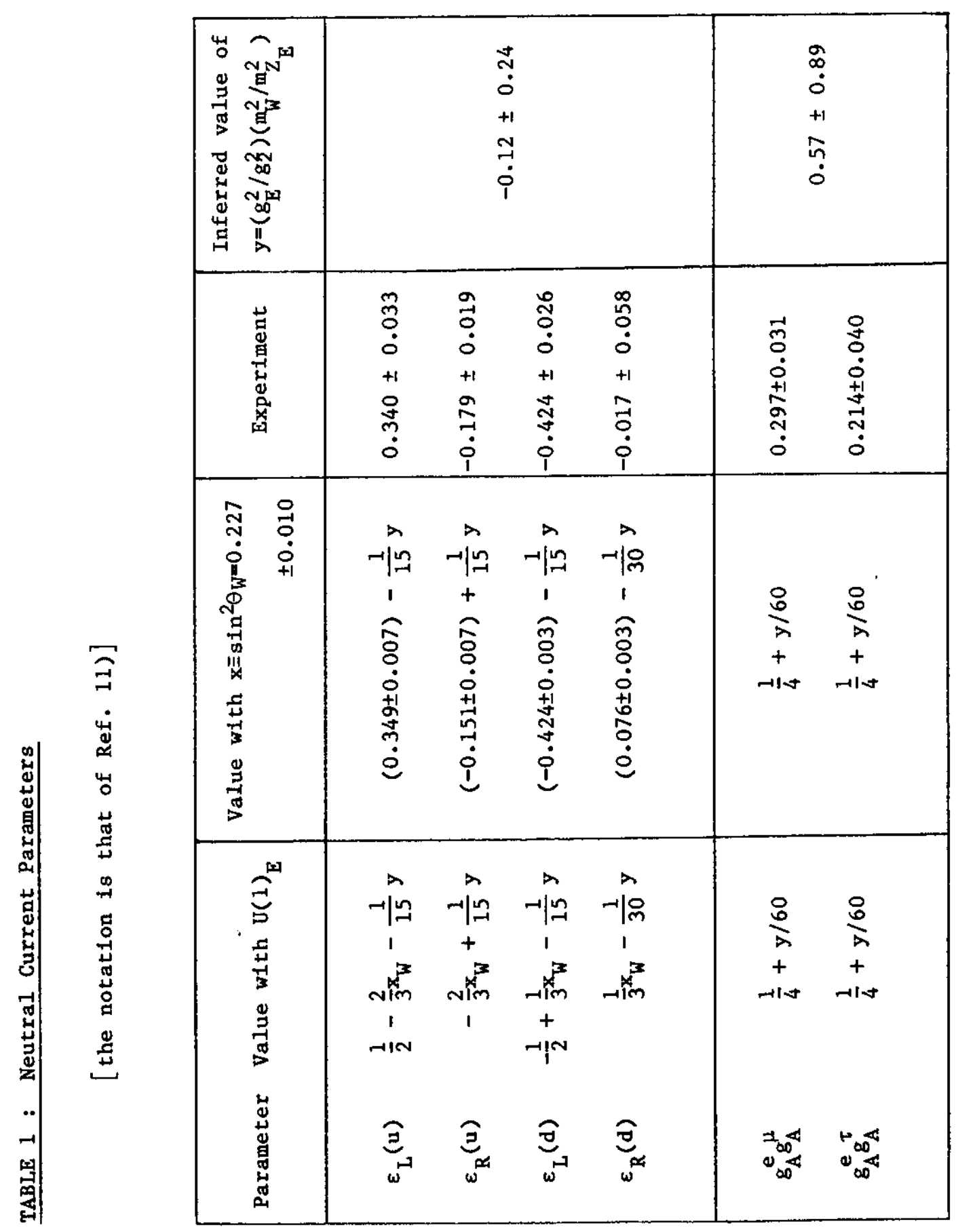


TABLE 2 : Supersymmetry Breaking Parameters

The parameters $A, A_{t}$ have the dimensions of mass. They and all the other mass parameters are expressed in units of $m_{\frac{1}{2}}$. These values were obtained with the representative inputs $\lambda_{t}^{2}\left(m_{W}\right)=0.12, \lambda^{2}\left(m_{W}\right)=0.078$

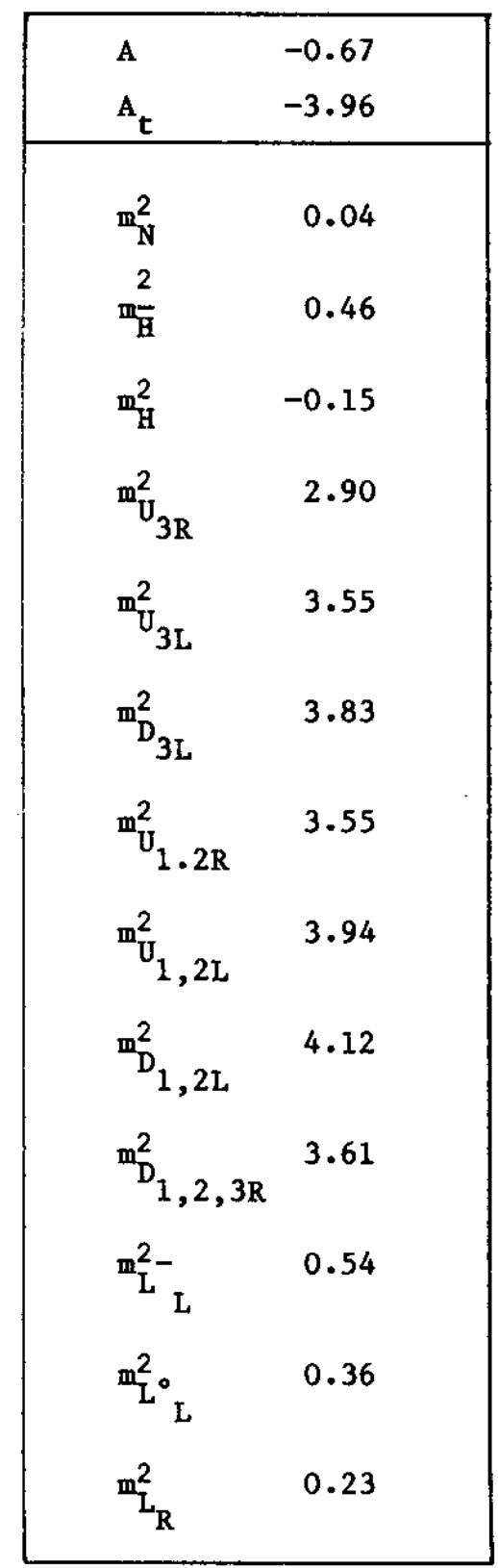




\section{R E F E R E N C E S}

1) M.B. Green, Surv.High Energy Phys. 3 (1983) 127;

J.H. Schwarz, Phys.Reports 89 (1982) 223.

2) M.B. Green and J.H. Schwarz, Phys.Letters 149B (1984) 117.

3) E. Witten, Phys.Letters 155B (1985) 151.

4) E. Cremmer, S. Ferrara, C. Kounnas and D.V. Nanopoulos, Phys.Letters 133B (1983) 61.

5) J. E111s, A.B. Lahanas, D.V. Nanopoulos and K. Tamvakis, Phys.Letters 134B (1984) 429;

J. Ellis, C. Kounnas and D.V. Nanopoulos, Nuclear Phys. B241 (1984) 406; Nuclear Phys. B247 (1984) 373 and Phys.Letters 143B (1984) 410.

6) E. Witten, Nuclear Phys. B258 (1985) 75;

S. Cecotti, J.-P. Derendinger, S. Ferrara, L. Girardello and M. Roncadelli, CERN preprint TH.4103 (1985);

J.D. Breit, B.A. Ovrut and G. Segrè, Phys.Letters 158B (1985) 33;

M. Dine, V. Kaplunovsky, M. Mangano, C. Nappi and N. Seiberg, Princeton preprint (1985);

M. Mangano, Princeton preprint (1985).

7) P. Candelas, G.T. Horowitz, A. Stromlnger and E. Witten, Nuclear Phys. B258 (1985) 46;

A. Strominger and E. Witten, Princeton preprint (1985).

8) J.-P. Derendinger, L.E. Ibañez and H.-P. Nilles, Phys.Letters 155B (1985) 65 ;

M. Dine, R. Rohm, N. Seiberg and E. Witten, Phys.Letters 156B (1985) 55.

9) E. Cohen, J. E111s, C. Gomez and D.V. Nanopoulos, CERN preprint TH.4159 (1985).

10) E. Cohen, J. Ellis, K. Enqvist and D.V. Nanopoulos, CERN preprint TH.4195 (1985).

11) J.E. Kim, P. Langacker, M. Levine and H.M. Will1ams, Rev.Mod.Phys. 53 (1981) 211.

12) R. Marsha11, Review presented at the 16th International Symposium on Multiparticle Dynamics, Kiryat Anavim, Israel (1985).

13) UA2 Collaboration, L. Mape11i, presented at the 5th Topical Workshop on $\overrightarrow{p p}$ Collider Physics, Saint Vincent, Aosta, CERN preprint EP/85-84 (1985).

14) K. Inoue, A. Kakuto, H. Komatsu and S. Takeshita, Progr.Theor.Phys. 68 (1982) 927 and 71 (1984) 413.

15) JADE Collaboration, W. Bartel et a1., Phys.Letters 155B (1985) 385, 392;

Mark J Collaboration, B. Adeva et a1., Phys.Letters 155B (1985) 439. 
16) UA1 Collaboration, G. Arnison et al., Phys.Letters 139B (1984) 115;

J. E1lis and H. Kowalski, Phys.Letters 142B (1984) 441 and CERN preprint TH. 4126 (1985);

E. Reya and D.P. Roy, Phys.Rev.Letters 52 (1984) 881.

17) P. Jennt, private communication (1985).

18) MAC Collaboration, E. Fernandez et a1., Phys.Rev.Letters 54 (1985) 1118.

19) S. Coleman and E. Welnberg, Phys.Rev. D7 (1973) 1888.

20) J.D. Breit, B.A. Ovrut and G. Segrè, University of Pennsylvania preprint UPR-0282T (1985);

P. BInétruy and M.K. Galllard, Berkeley preprint in preparation (1985).

21) M. Dine and N. Seiberg, Princeton preprints (1985). 